\title{
Physician-assisted death in Canada: an imminent reality
}

\author{
Ian A. Herrick, MD
}

Received: 12 November 2015/Accepted: 16 December 2015/Published online: 6 January 2016

(C) Canadian Anesthesiologists' Society 2016

On February 6, 2015, the Supreme Court of Canada unanimously declared the criminal prohibition against physician-assisted dying unconstitutional. ${ }^{1}$ In this landmark case, Carter v. Canada, the Supreme Court suspended the decision for 12 months to provide the federal and provincial governments, as well as medical regulatory authorities and associations, respectively, time to establish a legislative framework and to develop appropriate policies and guidelines. In its judgment, the court set out general criteria noting that individuals eligible for physicianassisted death (PAD) must be competent, consenting adults who are suffering a "grievous, irremediable medical condition" (involving an illness, disease, or disability) that they find intolerable. On February 6, 2016, the Criminal Code provisions banning assisted dying will terminate and physician-assisted suicide and euthanasia will cease to be illegal in Canada.

This month in the Journal, Mottiar et al. explore the implications of legalizing PAD in Canada and focus on issues particularly relevant to anesthesiologists. ${ }^{2}$ The discussion is both relevant and timely as the issue is emotionally charged, contentious, and intensely personal, with physicians - and the public at large - deeply divided on the issue. Many of the authors' concerns have been voiced by others, particularly the Canadian Medical Association (CMA), which has been at the centre of the national dialogue on this issue for several years. Part of the challenge associated with offering informed commentary on the issue of PAD in Canada is that there is currently

I. A. Herrick, MD ( $\square)$

Department of Anesthesia and Perioperative Medicine, Schulich

School of Medicine and Dentistry, Western University, 1151

Richmond Street, London, ON N6A 3K7, Canada

e-mail: iherrick@uwo.ca little established experience upon which to draw. As a consequence, commentators such as Mottiar et al. have little substance upon which to found their discussion, and they are left to speculate on what future policy choices might look like or to reflect on how experience in other jurisdictions might influence policy choices in Canada.

Canadians have recently elected a new federal government. The previous government under Prime Minister Stephen Harper opposed all legal challenges to the criminal code prohibition on PAD and chose to defer addressing the Supreme Court decision that struck down these laws until after the election held on October 19, 2015. It is not clear at this time what the newly elected government under Prime Minister Justin Trudeau will do with respect to the Supreme Court decision. Indeed, Justice Minister Jody Wilson-Raybould, along with other members of the new federal cabinet, was announced merely days before this editorial was written. At the time of the Supreme Court ruling in February 2015, Mr. Trudeau commented, "But if we do nothing, if we don't get this important national debate underway soon, Canada will find itself without any laws governing physician-assisted death. That kind of legislative vacuum serves no one - not people who are suffering, not their anxious family members, not the compassionate physicians who offer them care." 3 But the timeline for this legal change draws perilously short, and as Mottiar et al. emphasize, the federal government is not compelled to regulate PAD. In the absence of federal regulation, the provincial governments and provincial regulatory colleges will develop independent approaches to fill the void.

Although the issue was not discussed by Mottiar et al., federal engagement in this issue assumes additional urgency as the province of Quebec progresses independently toward implementation of Bill $52-A n$ Act 
respecting end of life care, which "prescribes the criteria that must be met for a person to obtain medical aid in dying" ${ }^{4}$ Supported by a large majority of the members of Quebec's National Assembly on a free vote held in June 2014, the Bill comes into force on December 10, 2015. It addresses a variety of end-of-life issues, including access to palliative care and access to physician-assisted death when requested by eligible, mentally competent adult patients who "suffer from an incurable serious illness; suffer from an advanced state of irreversible decline in capability; and suffer from constant and unbearable physical or psychological pain which cannot be relieved in a manner the person deems tolerable." As a consequence, Quebec will become the first province in Canada to allow competent adults suffering intolerably from an incurable illness to request medical aid in dying, although as noted, the Criminal Code prohibition on PAD will remain in force nationally until February 2016. It is not clear at this time how this apparent incongruence between federal law and provincial legislation will be resolved. In other respects, Quebec appears to be progressing on schedule with implementation. The Collège des médecins du Québec is reported to have developed a regulatory framework for PAD and, together with other regulators, including the Order of Pharmacists of Quebec and the Order of Nurses of Quebec, has established guidelines for physicians and other healthcare professionals engaged in PAD (available to members through the College website). ${ }^{5}$

Mottiar et al. legitimately probe a number of issues and questions of concern to the medical community generally as well as a few that apply to anesthesiologists specifically. Issues of general concern to the profession include the question whether regulations will include a duty to refer. This would require physicians who choose not to participate in PAD, as a matter of conscience, to refer eligible patients who request PAD - a hotly contested issue among physicians, the public, as well as medical regulators. Anesthesia-specific concerns include the question whether specialized knowledge and skills (such as pharmacological experience with drugs commonly used for PAD and procedural skills for establishing venous access, etc.) will promote the perception that anesthesiologists are well-suited to play prominent roles in PAD.

In addressing questions pertaining to duty to refer, Mottiar et al. note that the Supreme Court commented that nothing in its decision would compel physicians, as a matter of conscience, to provide assistance in dying, but it was silent on the issue of duty to refer. The authors suggest that, in the absence of legal or regulatory guidance to the contrary, current provincial college regulations that generally support a duty to refer seem to apply. While there is some variation in regulatory responses to this issue in other jurisdictions where PAD is available, many maintain an obligation for physicians who do not participate as a matter of conscience to refer patients eligible for PAD. ${ }^{6,7}$ It may provide some reassurance, should experience in Quebec serve as a national template, that Bill 52 has tempered this obligation by ensuring that continuity of care is maintained through referral to the executive director of the institution or local authority who will arrange for the transfer of care.

With respect to the role of the anesthesiologist in PAD, Mottiar et al. note that the sparse literature is unclear on the degree to which anesthesiologists are engaged in PAD in jurisdictions where it is available. There is also little evidence to suggest that anesthesiologists are treated differently than any other medical specialty with respect to a role in providing PAD. ${ }^{6,7}$ As a consequence, the reference by Mottiar et al. to attempts to recruit anesthesiologists to participate in capital punishment - an emotionally charged and arguably ethically unrelated practice - in some jurisdictions where it is sanctioned, does appear largely provocative.

The CMA has been a prominent voice for physicians in the national debate on PAD that precedes the Supreme Court decision. The CMA has taken a very thoughtful and principled approach to the issue on behalf of physicians. This action plan, to date, includes conducting surveys and town hall meetings across the country to gauge public and professional opinion on the issue $;^{8}$ amending the CMA policy on Euthanasia and Assisted Death ${ }^{9}$ to reflect changing attitudes; developing a draft policy framework for PAD to articulate foundational principles and promote national dialogue; ${ }^{6}$ and emphasizing that legislative responses to this issue should not occur in isolation from the obligation to ensure that patients across the country with irremediable conditions have access to appropriate health and supportive care, including timely access to palliative care services for those who need them. ${ }^{8}$

The Canadian Anesthesiologists' Society (CAS), through its Ethics Committee, has been following legal and legislative progress in relation to PAD in Canada for the past two years. The issue was brought to the CAS Board of Directors for information and discussion in June 2015. The CAS has deferred articulating an independent position with respect to $\mathrm{PAD}$, acknowledging the enormous work done by the CMA on behalf of Canadian physicians in general, including anesthesiologists. The CAS Ethics Committee is currently organizing an ethics symposium for the 2016 annual meeting in Vancouver on the topic of physician-assisted death. Discussion will potentially include newly established national and/or provincial legislative responses to the Supreme Court decision legalizing PAD and may be informed by experience in Quebec as the first jurisdiction in Canada to provide 
medical aid in dying. The journey will be challenging and answers for many questions, at least in the Canadian context, remain unclear. Mottiar et al. cast light on many relevant questions and encourage Canadian anesthesiologists to stay informed regarding the evolving legislative and regulatory environment surrounding PAD. As noted by Dr. Jeff Blackmer, CMA Vice-President, Medical Professionalism, in relation to the status of physician-assisted death in Canada, "Legalization of assisted dying is a societal prerogative. It is a done deal. But we still have an opportunity to help shape what it will look like in practice." 8

\section{L'aide médicale à mourir au Canada: une réalité imminente}

Le 6 février 2015, la Cour suprême du Canada a, à l'unanimité, déclaré inconstitutionnelle la prohibition criminelle de l'aide médicale à mourir. ${ }^{1}$ Dans cette cause qui fait jurisprudence, Carter c. Canada, la Cour suprême a suspendu sa décision pour une période de 12 mois afin de donner aux gouvernements fédéral et provinciaux, ainsi qu'aux Ordres et associations de médecins, respectivement, le temps de mettre en place un cadre législatif et de mettre au point des politiques et des directives adaptées. Dans son jugement, la Cour a présenté des critères généraux selon lesquels les personnes éligibles à l'aide médicale à mourir (AMM) devaient être des adultes compétents et consentants souffrant de "problèmes de santé graves et irrémédiables » (y compris une affection, une maladie ou un handicap) qui leur sont intolérables. Le 6 février 2016, les provisions du Code criminel prohibant l'aide à mourir deviendront caduques, et l'aide au suicide et l'euthanasie ne seront plus illégales au Canada.

Dans ce numéro du Journal, Mottiar et coll. explorent les implications d'une légalisation de l'AMM au Canada et se concentrent sur les questions particulièrement brûlantes pour les anesthésiologistes. ${ }^{2}$ Cette discussion arrive à point nommé et est plus que pertinente. En effet, la question de l'AMM est très chargée émotionnellement, sujette à controverse et très personnelle, et elle divise profondément, tant les médecins que le grand public. Bon nombre des inquiétudes des auteurs ont été exprimées par d'autres, notamment par l'Association médicale canadienne (AMC), qui est au cœur du dialogue national sur cette question depuis plusieurs années. Il est difficile de proposer un commentaire éclairé sur la question de l'AMM au Canada, en partie parce que nous n'avons, à l'heure actuelle, que peu d'expériences sur lesquelles nous appuyer. Dès lors, les commentateurs tels que Mottiar et coll. ne disposent que d'une quantité limitée de matériel pour étayer leur discussion, et il ne leur reste qu'a spéculer sur les choix de politiques futures ou à réfléchir à la façon dont les expériences vécues dans d'autres territoires pourraient avoir un impact sur nos politiques ici, au Canada.

Les Canadiens viennent d'élire un nouveau gouvernement fédéral. Le gouvernement précédent, sous le premier ministre Stephen Harper, s'opposait à toute contestation judiciaire de la prohibition du code criminel sur l'AMM et a choisi de différer toute prise de position concernant la décision de la Cour suprême invalidant ces lois jusqu'à la tenue des élections le 19 octobre 2015. À l'heure actuelle, nous ne savons pas ce que le gouvernement nouvellement élu, sous le premier ministre Justin Trudeau, fera quant à la décision de la Cour suprême. En effet, la nomination de Jody Wilson-Raybould au poste de ministre de la Justice ainsi que des autres membres du nouveau Cabinet fédéral n'a été annoncée que quelques jours avant la rédaction de cet éditorial. Au moment de la décision de la Cour suprême en février 2015, M. Trudeau disait : « [Mais] si nous ne faisons rien, si nous n'entamons pas bientôt ce débat national important, le Canada ne disposera d'aucune loi pour gouverner l'aide médicale à mourir. Ce type de vide législatif n'est dans l'intérêt de personne : ni des gens qui souffrent, ni des membres de leur famille qui s'inquiètent pour eux, ni des médecins compatissants qui les soignent. ${ }^{3}$ Mais le délai avant que la loi ne soit amendée devient dangereusement court et, comme le soulignent Mottiar et coll., le gouvernement fédéral n'est pas contraint de réglementer l'AMM. En l'absence d'un règlement fédéral, les gouvernements provinciaux et les organismes de réglementation provinciaux mettront au point des approches indépendantes pour combler ce vide.

Bien que Mottiar et coll. n'abordent pas cette question, l'implication fédérale se fait encore plus urgente au fur et à mesure que le Québec progresse, de façon indépendante, vers la mise en œuvre de la Loi 52 - une Loi concernant les soins de fin de vie, qui «prescrit [...] les conditions permettant à une personne d'obtenir l'aide médicale à mourir ». ${ }^{4}$ Appuyée par une importante majorité des membres de l'Assemblée nationale du Québec lors d'un vote libre tenu en juin 2014, la Loi est prévue d'entrer en vigueur le 10 décembre 2015. Elle aborde diverses questions liées à la fin de vie, notamment l'accès aux soins palliatifs et à l'aide médicale à mourir lorsqu'il est exigé par un patient majeur éligible, apte mentalement et qui « est [atteint] d'une maladie grave et incurable; [lorsque] sa situation médicale se caractérise par un déclin avancé et irréversible de ses capacités; [qu'il] éprouve des souffrances physiques ou psychiques constantes, insupportables et qui ne peuvent être apaisées 
dans des conditions [qu'il] juge tolérables. » Le Québec pourrait donc devenir la première province canadienne à autoriser les adultes aptes et endurant des souffrances intenables provoquées par une maladie incurable à demander une aide médicale à mourir bien que, comme nous l'avons mentionné, le Code criminel interdise l'AMM jusqu'en février 2016. Nous ne savons pas, au moment de rédiger cet éditorial, comment cette inadéquation apparente entre la loi fédérale et la législation provinciale sera traitée. À d'autres égards, le Québec semble progresser selon son calendrier de mise en œuvre. Le Collège des médecins du Québec a mis au point un cadre législatif pour l'AMM et, de concert avec d'autres organismes de réglementation, notamment l'Ordre des pharmaciens du Québec et l'Ordre des infirmières et des infirmiers du Québec, établi des directives destinées aux médecins et autres professionnels de la santé concernés par l'AMM (le document est disponible pour les membres sur le site Internet du Collège) ${ }^{5}$

De façon légitime, Mottiar et coll. examinent plusieurs questions et préoccupations touchant la communauté médicale en général, ainsi que quelques-unes qui sont propres aux anesthésiologistes. Les préoccupations qui touchent toute la profession concernent notamment la question de savoir si les lois incluront un 'devoir de référer' (duty to refer). Un tel acte obligerait les médecins qui décident de ne pas participer à l'AMM, pour des raisons de conscience, de référer les patients éligibles qui demandent une AMM. Cette question est très controversée tant parmi les médecins, le grand public que les législateurs médicaux. Les inquiétudes touchant spécifiquement l'anesthésie comprennent notamment la question de savoir si des connaissances et des compétences spécialisées (comme l'expérience pharmacologique avec les médicaments fréquemment utilisés pour l'AMM et les compétences procédurales pour établir un accès veineux, etc.) donneront l'impression que les anesthésiologistes sont bien placés pour jouer un rôle proéminent dans l'AMM.

En abordant certaines des questions liées au devoir de référer, Mottiar et coll. notent que la Cour suprême a commenté que rien, dans sa décision, n'obligerait les médecins, pour une question de conscience, de procurer une aide à mourir, mais que la Cour n'a pas statué sur le devoir de référer. Les auteurs suggèrent que, en l'absence de cadre légal ou réglementaire spécifiant le contraire, la réglementation actuelle des collèges provinciaux, qui est en général en faveur du devoir de référer, semble s'appliquer. Bien que la loi varie en ce qui touche à cette question dans les territoires où l'AMM est déjà disponible, dans bon nombre de cas les médecins qui ne souhaitent pas participer à l'AMM pour des raisons de conscience ont le devoir de référer les patients éligibles. ${ }^{6,7} \mathrm{Si}$ l'on se fonde sur ce qui va se faire au Québec, cela pourrait nous rassurer à l'échelle nationale. En effet, la Loi 52 a tempéré cette obligation en s'assurant que la continuité des soins soit maintenue en référant le patient au directeur général de l'institution ou aux autorités locales, qui s'occuperont alors du transfert de soins.

En ce qui touche au rôle de l'anesthésiologiste dans l'AMM, Mottiar et coll. remarquent que le peu de littérature disponible à ce sujet est peu claire quant à la mesure dans laquelle les anesthésiologistes sont impliqués dans l'AMM là où elle est disponible. De plus, rien ne laisse à penser que les anesthésiologistes soient traités différemment de toute autre spécialité médicale en ce qui a trait à leur rôle pour la fourniture de l'AMM..${ }^{6,7}$ Dès lors, l'analogie de Mottiar et coll. avec des tentatives de recrutement d'anesthésiologistes pour participer à la peine de mort, une pratique très chargée émotionnellement et probablement sans lien éthique quelconque à l'AMM, dans certains des territoires où elle est en vigueur, semble avoir pour unique but de provoquer le lecteur.

L'AMC a joué un rôle important pour défendre les intérêts des médecins dans le débat national sur l'AMM qui a précédé la décision de la Cour suprême. Au nom des médecins, l'AMC a adopté une approche très réfléchie et fondée sur des principes clairs de la question. À ce jour, le plan d'action comprend la réalisation de sondages et de réunions citoyennes partout au pays pour évaluer les opinions du public et des professionnels quant à cette question délicate ${ }^{8}$ la modification de la politique de l'AMC sur l'euthanasie et l'aide à mourir' pour refléter les changements d'attitude; l'élaboration d'une ébauche de cadre de politique pour l'AMM afin d'en énoncer les principes fondateurs et de promouvoir un dialogue à l'échelle nationale ${ }^{6}{ }^{6}$ et le rappel que les réponses juridiques à cette question ne devraient pas être émises sans tenir compte de l'obligation de garantir que les patients partout au pays souffrant de maladies incurables aient accès à des soins de santé et de soutien adaptés, y compris un accès à des services de soins palliatifs pour ceux qui en ont besoin, quand ils en ont besoin. ${ }^{8}$

La Société canadienne des anesthésiologistes (SCA), par l'entremise de son Comité de déontologie, suit depuis deux ans les progrès légaux et législatifs concernant l'AMM au Canada. La question a été portée à l'attention du Conseil d'administration de la SCA à des fins d'information et de discussion en juin 2015. La SCA a choisi de ne pas articuler de position indépendante concernant l'AMM tout de suite, reconnaissant ainsi le travail gigantesque réalisé par l'AMC au nom des médecins canadiens en général, y compris des anesthésiologistes. Le Comité de déontologie de la SCA travaille actuellement à la mise au point d'un symposium sur l'éthique qui se tiendra dans le cadre du Congrès annuel 2016 à Vancouver sur le thème de l'aide 
médicale à mourir. Les discussions pourraient porter sur les réponses législatives nationales et/ou provinciales nouvellement établies suite à la décision de la Cour suprême légalisant l'AMM et pourraient tirer parti de l'expérience québécoise, cette province étant la première à proposer une aide médicale à mourir au Canada. Le voyage sera difficile et les réponses à bon nombre de questions, pour le moins dans le contexte canadien, demeurent floues. Mottiar et coll. mettent en lumière plusieurs questions pertinentes et encouragent les anesthésiologistes canadiens à s'informer régulièrement quant à l'environnement législatif et réglementaire en pleine évolution entourant l'AMM. Comme le note le Dr Jeff Blackmer, vice-président de la section du Professionnalisme médical de l'AMC, concernant le statut de l'aide médicale à mourir au Canada, « La légalisation de l'aide à mourir est une prérogative sociétale. C'est un fait accompli. Mais nous avons encore l'occasion de contribuer à la forme qu'elle prendra en pratique. $»^{8}$

Conflicts of interest None declared.

Conflit d'intérêt Aucun.

\section{References}

1. Judgments of the Supreme Court of Canada. Carter v. Canada (Attorney General) 2015 SCC 5, 1 SCR 331. Available from URL: http://scc-csc.lexum.com/scc-csc/scc-csc/en/item/14637/index.do (accessed November 2015).
2. Mottiar M, Grant C, McVey MJ. Physician-assisted death and the anesthesiologist. Can J Anesth 2016; 63: DOI:10.1007/s12630015-0571-0.

3. Trudeau J. Remarks by Justin Trudeau on the Liberal Opposition Day Motion on Physician-Assisted Dying. February 25, 2015. Available from URL: http://www.liberal.ca/remarks-by-justintrudeau-on-physician-assisted-dying/ (accessed November 2015).

4. National Assembly. Bill No 52: An Act Respecting End-of-Life Care - 2013. Available from URL: http://www.assnat.qc.ca/en/ travaux-parlementaires/projets-loi/projet-loi-52-40-1.html (accessed November 2015).

5. Kirkey S. Quebec doctors to get standard euthanasia kits as province prepares to legalize 'medical aid in dying'. Montreal Gazette, August 27, 2015. Available from URL: http://www. montrealgazette.com/health/qubec+doctors+standard+euthanasi a+kits+province+prepares+legalize/11330747/story.html (accessed November 2015).

6. Canadian Medical Association. Schedule A - Principles-Based Approach to Assisted Dying in Canada (Backgrounder) - Supreme Court Decision in "Carter" Physician-Assisted Death Case (draft) Available from URL: https://www.cma.ca/Assets/assets-library/ document/en/advocacy/EOL/care-at-the-end-of-life-cma-frameworkjune2015-e.pdf (accessed November 2015).

7. Canadian Medical Association. Schedule A: Legal Status of Physician Assisted Death (PAD) in Jurisdictions with Legislation. Available from URL: https://www.cma.ca/Assets/assets-library/ document/en/advocacy/EOL/Legal-status-physician-assisted-deathjurisdictions-legislation.pdf (accessed November 2015).

8. Canadian Medical Association. A Canadian Approach to Assisted Dying: CMA Member Dialogue. Summary Report. Available from URL: https://www.cma.ca/Assets/assets-library/document/ en/advocacy/Canadian-Approach-Assisted-Dying-e.pdf (accessed November 2015).

9. Canadian Medical Association. CMA Policy: Euthanasia and Assisted Death (Update 2014). Available from URL: https://www. cma.ca/Assets/assets-library/document/en/advocacy/EOL/CMA Policy_Euthanasia_Assisted\%20Death_PD15-02-e.pdf (accessed November 2015). 